\section{A E E T}

ASOCIACIÓN ESPAÑOLA DE ECOLOGÍA TERRESTRE

\title{
Reflexiones sobre el impacto y manejo de la caza de mamíferos silvestres en los bosques tropicales
}

\author{
J.E. Fa1,2,*, M.A. Farfán ${ }^{3,4}$, A.L. Márquez ${ }^{4}$, J. Duarte ${ }^{3,4}$, J.M. Vargas ${ }^{4}$ \\ (1) Durrell Wildlife Conservation Trust, Les Augrès Manor, Trinity, Jersey JE3 5BP, UK. \\ (2) ICCS, Division of Biology, Imperial College London, Silwood Park Campus, Buckhurst Road, Ascot SL5 7PY, UK. \\ (3) Biogea Consultores, Calle Navarro Ledesma 243 P4-3ㄷ 29010 - Málaga, España. \\ (4) Departamento de Biología Animal, Facultad de Ciencias, Universidad de Málaga, E-29071 Málaga, España.
}

*Autor de correspondencia: J.E. Fa [John.Fa@durrell.org]

> Recibido el 21 de febrerode 2013, aceptado el 26 de mayo de 2013.

Fa, J.E., Farfán, M.A., Márquez, A.L., Duarte, J., Vargas, J.M. (2013). Reflexiones sobre el impacto y manejo de la caza de mamíferos silvestres en los bosques tropicales. Ecosistemas 22(2):76-83. Doi.: 10.7818/ECOS.2013.22-2.12

Los bosques tropicales de todo el mundo proveen productos renovables (maderables y de otra naturaleza) a sus numerosos habitantes. Para muchas comunidades autóctonas de las selvas en América, África y Asia, la fauna es una fuente importante de proteína animal y también de ingresos derivados de la venta de los animales cazados. La caza comercial de fauna selvática, junto con las demandas impuestas por el intenso crecimiento de la población humana en estos ecosistemas, supone sin embargo una amenaza para la viabilidad de muchas poblaciones de mamíferos silvestres. Este impacto pone en riesgo la biodiversidad de estos ecosistemas y puede reducir las fuentes de proteínas para las poblaciones rurales. En este trabajo se presenta un análisis del volumen de carne de monte extraída en selvas tropicales a escala mundial, y se examina su importancia en las dietas y economías de las comunidades rurales y urbanas en estas regiones. Se realiza una breve revisión sobre el estado de conservación de aquellas especies afectadas por la caza, y de los procesos que han contribuido a su situación actual. Se concluye con un análisis de las soluciones propuestas para minimizar el conflicto entre la protección de la biodiversidad y las necesidades de las poblaciones humanas dependientes de ella en las selvas tropicales.

Palabras clave: África, amenazas, Asia, caza comercial, caza de subsistencia, Suramérica

Fa, J.E., Farfán, M.A., Márquez, A.L., Duarte, J., Vargas, J.M. (2013). Reflections on the impact and management of hunting of wild mammals in tropical forests. Ecosistemas 22(2):76-83. Doi.: 10.7818/ECOS.2013.22-2.12

Globally, tropical forests provide renewable products (timber and other non-timber goods) to its many inhabitants. For many indigenous communities in the forests of South America, Africa and Asia, wildlife and more particularly the meat from mammals, is an important source of animal protein, but also a source of income derived from the sale of the hunted animals. Commercial hunting of jungle fauna, together with the demands imposed by the high population increase in these ecosystems, threatens the viability of many populations of wild mammals. This impact puts at risk many mammalian communities and reduces the availability of protein for rural populations. This paper presents an analysis of bushmeat species extracted in tropical forests globally, and discusses their importance in diets and economies of rural and urban communities in these regions. We review the state of conservation of those species affected by hunting, and of the processes that have contributed to the current situation. We conclude with an analysis of solutions that could minimize the conflict between the protection of biodiversity and the needs of human populations dependent on it in the tropical jungles.

Key words: Africa, Asia, commercial hunting, South America, subsistence hunting, threats

\section{Introducción}

En las zonas de selva lluviosa del planeta la carne de los animales salvajes ha constituido, desde la prehistoria, un componente básico de la dieta de sus habitantes. En contra de la visión popular, esta preferencia cultural no es consecuencia de una secular falta de conciencia o de una más reciente explotación comercial. En los últimos tiempos la caza utilitaria de animales silvestres se relaciona con la falta de alternativas proteicas, ya que existe una baja productividad del ganado doméstico en los bosques tropicales a raíz de los elevados riesgos y costes de inversión asociados a su cría. Además, el escaso poder adquisitivo de la mayoría de los habitantes de las zonas forestales, las peculiaridades del clima tropical y las enfermedades asociadas impiden que la cría extensiva de ganado constituya una opción viable. En consecuencia, la caza silvestre constituye la forma más accesible y fácil de obtener proteína animal, por lo que representa la principal fuente de abastecimiento para numerosas familias en zonas rurales y también para muchas familias urbanas (Chardonnet et al. 1995). Paralelamente, la carne de caza constituye una importante fuente de ingresos para los cazadores e intermediarios que canalizan su venta.

En los últimos años se ha intensificado la caza comercial, debido a la demanda de alimentos generada por el incremento demográfico humano, la modernización de las técnicas cinegéticas y la mayor accesibilidad a zonas remotas (Redford y Robinson 1987; Wilkie y Carpenter 1999). Ello ha provocado un aumento de 
la presión sobre determinadas especies animales, especialmente las de mayor tamaño, provocando en algunos casos su extinción local por ser más vulnerables a la sobreexplotación (Peres 2000; Redford 1992). La información empírica disponible confirma que la caza comercial ha crecido en importancia desde hace algún tiempo (Hart 2000), así como el número de cazadores que suplementan sus ingresos con la venta de la carne. Esto se ha traducido en tasas de extracción que reducen las probabilidades de aprovechamiento sostenible de numerosas especies silvestres (Bennett y Robinson 2000). Este mismo fenómeno también ocurre en ecosistemas acuáticos. De hecho, estudios basados en los datos pesqueros han puesto de manifiesto la aparición del efecto "deterioro de las redes tróficas marinas", fenómeno que ha tenido lugar desde la llegada de la pesca industrial (Pauly y Palomares 2005). Consiste en una reorientación de las capturas hacia las especies de menor tamaño, en un determinado ecosistema, una vez agotadas las grandes especies localizadas en la cúspide de la cadena trófica.

En los bosques tropicales, las especies cazadas/recolectadas abarcan una amplia gama de tamaños, desde insectos hasta elefantes. El grupo de vertebrados capturado con más frecuencia, y que aporta mayor biomasa de carne para consumo propio o con una finalidad comercial, son los mamíferos (Fig. 1). Varios estudios indican que más del $90 \%$ de las especies extraídas de los bosques tropicales son, por orden de importancia, ungulados, roedores y primates (Robinson y Bennett 2004; Fa et al. 2006). Junto a las amenazas derivadas de la pérdida de hábitat y la deforestación, la extinción global de muchas especies de mamíferos, especialmente las más sensibles (primates, grandes ungulados o paquidermos), podría producirse debido a un cúmulo de desapariciones locales provocadas por la creciente presión cinegética (Fa y Brown 2009). A largo plazo ello podría ocasionar el empobrecimiento de los bosques (creando el síndrome del 'bosque vacío' de Redford, 1992), y cambios en la dinámica y estructura de las masas forestales, debido a la pérdida de especies dispersoras de semillas y de animales "ingenieros del ecosistema" tales como los elefantes.

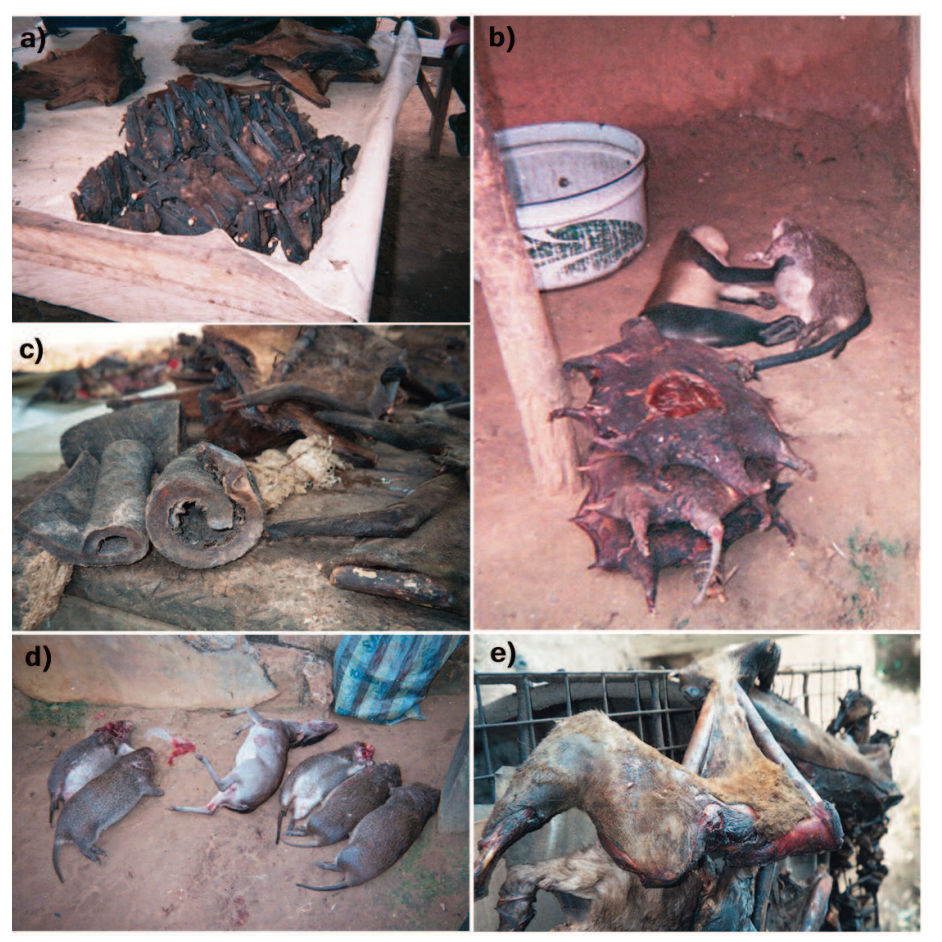

Figura 1. Ejemplos de individuos ahumados o frescos de algunas especies de mamíferos vendidas en mercados de carne de caza en África: a) murciélagos (Eidolon helvum); b) pangolínes (Phataginus tricuspis), puercoespín (Atherurus africanus) y mono (Cercopithecus mona); c) piel de elefante de bosque (Loxodonta cyclotis); d) ratas de cañaveral (Thryonomys swinderianus) y duikero azul (Philantomba montícola); e) pata ahumada de mono dril (Mandrillus leucophaeus).
La pérdida de diversidad comenzó a observarse en los bosques de Asia, ya que en los últimos 40 años se han extinguido 12 especies de grandes mamíferos en Vietnam, debido en gran parte a la caza (Bennett y Rao 2002). El problema es actualmente más acuciante en las zonas tropicales del África occidental y central, aunque posiblemente en los próximos 10-20 años las extinciones se incrementarán en las regiones más remotas de Suramérica (Peres 2001). Este patrón es consecuencia de los impactos derivados del desarrollo desmedido y de la pérdida de hábitat en los tres continentes, ligados fundamentalmente al enorme crecimiento de la población humana: actualmente hay alrededor de 500 habitantes $/ \mathrm{km}^{2}$ en las zonas de bosques tropicales asiáticos, unos 100 habitantes $/ \mathrm{km}^{2}$ en los africanos y cerca de 50 habitantes $/ \mathrm{km}^{2}$ en Suramérica (Fa y Peres 2001).

A pesar de que la caza de mamíferos es práctica habitual en los tres continentes, la situación en Asia difiere de la de África o Suramérica ya que el comercio de fauna silvestre en la región asiática se realiza a gran escala. El este y sureste asiático constituyen el principal núcleo de consumo de subproductos de animales silvestres, tales como huesos de tigre por sus propiedades medicinales, aletas de tiburón con fines culinarios o cuernos de rinoceronte por sus cualidades afrodisíacas. La región es, además, un proveedor clave del mercado internacional de vida silvestre, tanto legal como ilegal. Sin embargo, en África y Suramérica la caza y el comercio de la carne es más local.

En este artículo se muestra una perspectiva general de la caza de mamíferos silvestres en las selvas tropicales de Suramérica, África y Asia. Asimismo, se revisa y sintetiza los resultados de investigaciones realizadas sobre el consumo de carne de caza, y su importancia para el bienestar humano y seguridad alimentaria en muchas zonas tropicales.

\section{Distribución y características de las selvas tropicales}

El bosque ombrófilo, o más específicamente el bosque húmedo tropical y subtropical, está estrechamente ligado a las zonas húmedas y templadas ecuatoriales, cuyas precipitaciones superan los $2000 \mathrm{~mm}$ anuales. Este tipo de bosques se caracteriza por la presencia de varios estratos de árboles perennifolios, con grandes hojas y anchas raíces a modo de contrafuertes que se ensanchan cerca del suelo. Las variaciones térmicas son inferiores a $5^{\circ} \mathrm{C}$ y las Iluvias persisten durante casi todo el año. Las selvas tropicales están presentes en el sureste asiático (desde Myanmar hasta Filipinas, Malasia, Indonesia, Papúa Nueva Guinea y noroeste de Australia), Sri Lanka, África subsahariana desde Camerún hasta el Congo (Selva del Congo), Suramérica (por ejemplo, la selva Amazónica), América Central (Bosawás, en el sur de la Península del Yucatán-El Petén-Belice-Calakmul), y muchas islas del Pacífico (tales como las Hawai). A pesar de su amplia distribución geográfica, existen grandes semejanzas florísticas (a nivel de familias y géneros) entre las regiones selváticas del mundo, lo que pone de manifiesto su origen común.

Los bosques de la Cuenca del Congo ocupan la mayor extensión de selva en África. La cuenca tiene forma de herradura y está formada por rocas sedimentarias que yacen sobre estratos del Precámbrico. La mayor parte de la cuenca tiene una altitud inferior a los $1000 \mathrm{~m}$ y muestra una topografía llana o ligeramente ondulada, con amplias zonas interiores pantanosas. El río Congo discurre por la zona oeste de la cuenca, dentro del Golfo de Guinea, a través de la planicie costera de Gabón. La región selvática más extensa del mundo se localiza en las cuencas de los ríos Amazonas y Orinoco. En general, los bosques amazónicos poseen un clima más húmedo que sus homólogos africanos. La gran extensión de la selva amazónica, junto con los diferentes tipos de suelos y la red fluvial interna de la Cuenca, permiten la existencia de diferentes tipos de bosque, cada uno de los cuales muestra un tipo característico de flora. La mayor parte de la región está ocupada por bosques sobre suelos bien drenados y con una alta disponibilidad de nutrientes. Otros tipos de bosques crecen en suelos más pobres o 
en zonas sujetas a incendios e inundaciones. Las selvas del lejano Oriente constituyen una unidad relativamente coherente, compartiendo numerosos elementos florísticos entre sus parches. Asimismo muestran una enorme semejanza florística con las zonas tropicales africanas, ya que ambas proceden de la fragmentación de la biota ancestral de Laurasia.

\section{Especies cazadas en las selvas tropicales}

La mayor parte de las especies de mamíferos con un peso corporal superior a los 1-2 kg es ampliamente cazada en los bosques tropicales (Tabla 1), mientras que la captura de animales de menor tamaño (principalmente roedores pequeños) está más restringida. Los Primates constituyen el grupo más diverso, seguidos por los Artiodáctilos, Roedores, Murciélagos y Carnívoros (Tabla 2).

A partir de la bibliografía consultada, se tiene constancia de que en las selvas tropicales se capturan hasta 470 especies de mamíferos, $18 \%$ de las cuales se encuentran amenazadas (25\% en Suramérica y $20 \%$ en África) (Tabla 2). Según Fa y Peres (2001) la proporción de taxones que se caza en el Amazonas (65\% de las 210 especies de mamíferos existentes) es superior a la detectada en la Cuenca del Congo (45 \% de 284).

En términos absolutos, la diversidad de especies capturadas en las selvas asiáticas es mayor que en los otros continentes. Dicho incremento se debe a la presencia de una amplia variedad de murciélagos frugívoros, muchos de ellos endémicos y restringidos a una sola isla de las existentes alrededor de Malasia, Filipinas e Indonesia. De hecho, se ha constatado la captura de más de 40 especies de Quirópteros Orientales. No obstante, la proporción total de especies amenazadas objeto de captura (12\%) es menor que en los otros dos grandes bloques selváticos del mundo.

Las estimas de extracción realizadas hasta ahora se han centrado en valoraciones globales de la proporción de proteínas que aportan a la dieta humana (Chardonnet et al. 1995), o en extrapolaciones del número de ejemplares y biomasa consumidos en regiones particulares. Aunque no se han realizado aún estimas fiables de la carne de mamíferos silvestres procedente de las selvas de Asia (ver Corlett 2007), se dispone de información actualizada para las cuencas del Congo y del Amazonas (Fa y Peres 2001; Fa et al. 2003). A partir de estos estudios, se calculó la tasa de extracción para 57 taxones de mamíferos, en una región forestal de 1.8 millones de $\mathrm{km}^{2}$ con una población rural de 24 millones de habitantes (Wilkie y Carpenter 1999).

Los cálculos realizados revelan que aproximadamente 579 millones de animales son anualmente consumidos en la cuenca del Congo, lo que representa unos 4 millones de toneladas de carne de caza (Fa et al. 2003). Este valor contrasta con el aportado por Wilkie y Carpenter (1999), que estimaron solamente un millón de toneladas basándose en extrapolaciones del consumo actual de carne (ver debajo). Pero estas cifras subestiman los valores reales, pues sólo tienen en cuenta una fracción de familias rurales e ignoran el consumo en los hogares urbanos. A pesar de este sesgo, la cantidad de carne de caza extraída y consumida por unidad de área en la cuenca del Congo es mayor que en el Amazonas. En términos de rendimiento neto, dado que la masa muscular y las vísceras comestibles representan aproximadamente el $55 \%$ de la masa corporal, 62808 toneladas son consumidas en el Amazonas (Peres 2000), mientras que en África dicha cifra asciende a unos dos millones de toneladas.

Al comparar las tasas de producción y extracción de mamíferos silvestres en la Amazonía y la cuenca del Congo, derivadas del estudio efectuado por Fa et al. (2002), se aprecia que en el Congo la mayoría de las especies está sometida a una explotación insostenible, mientras que en la Amazonía todavía se encuentra por debajo de dicho umbral (Fig. 2). De hecho, la relación entre ambas tasas es de $-23.37 \%$ para la Amazonía y $-7.59 \%$ para el Congo. Asimismo, 12 de las 17 especies de mamíferos africanos de gran tamaño $(>70 \%)$ se consideran sobreexplotadas, tales como todos los primates, elefantes, grandes ungulados y el búfalo de bosque
(Syncerus caffer nanus), ya que las tasas de extracción superan el $20 \%$ y rebasan el nivel de explotación máxima sostenible (Fa et al. 2002).

Estas diferencias intercontinentales son debidas, en gran parte, al mayor tamaño de la población humana y a la menor superficie forestal del Congo, y al hecho de que una gran proporción de las capturas es vendida en las ciudades y pueblos con una finalidad comercial. Por lo tanto, las tasas de captura per capita $(\mathrm{kg} / \mathrm{per}-$ sona/año) en relación al número de consumidores muestra una pequeña variación en los asentamientos de Suramérica, mientras que en África la media oscila entre $0,5 \mathrm{~kg} /$ persona/año en los poblados más pequeños y $1 / \mathrm{kg} /$ persona/año en los más grandes.

\section{Estimas del consumo de carne de mamíferos silvestres}

En la mayoría de las áreas selváticas donde se practica la caza, la biomasa extraída se destina al consumo local, ya sea por el propio cazador y su familia o vendida en los numerosos mercados que existen en distintos países, especialmente en África (Fa 2007; Dupain et al. 2012). Una parte de la diversidad cazada entra en redes comerciales internacionales, aunque de momento es sólo una pequeña fracción del total extraído en los países de origen. Sin embargo, el estudio de Chaber et al. (2010) apunta que hasta 200 toneladas de carne de caza, provenientes de países tropicales, pueden entrar a Francia en un año a través del aeropuerto Charles De Gaulle de Paris.

En Asia el comercio del cuerno y del marfil ha fomentado la caza de rinocerontes y elefantes (Rabinowitz 1995), pero la principal característica del actual comercio asiático de fauna y productos derivados es su amplitud y volumen (Corlett 2007). China ha sido durante mucho tiempo un importante mercado para productos animales de lujo, si bien durante los últimos 20 años se ha desarrollado una colosal industria de animales vivos y de algunas de sus partes, procedentes del sureste asiático (Li y Li 1998, Li y Wang 1998, Nooren y Claridge 2001). Ciertas especies se importan en calidad de alimento o por sus pieles, pero muchas otras se destinan a la medicina tradicional china. Este comercio ha sido impulsado por la creciente prosperidad económica del país y se ha visto favorecido por la mayor porosidad de sus fronteras, la disminución de la fauna autóctona de China y el cumplimiento más efectivo de las leyes que la protegen.

En la actualidad, los comerciantes de Asia y África utilizan una gran variedad de especies con diferentes propósitos (Nooren y Claridge 2001, Shepherd 2001). El bienestar urbano está sustituyendo progresivamente a la pobreza rural como motor de la caza

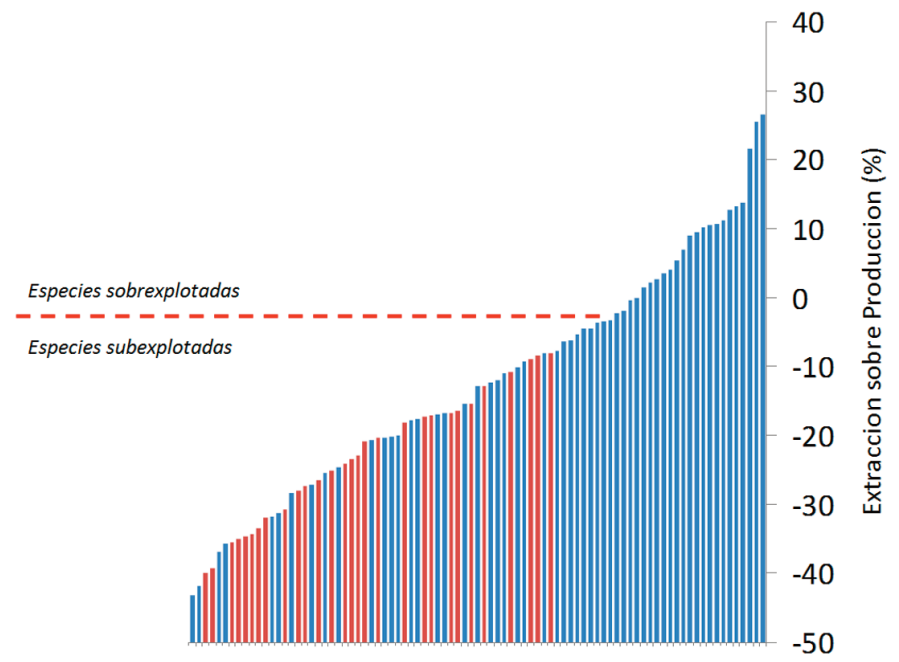

Figura 2. Relación entre las tasas de extracción y de producción de especies de mamíferos cazados en los bosques tropicales de la cuenca del Amazonas (barras rojas) y del Congo (barras azules). La línea punteada indica que la extracción es igual a la producción (datos de Fa et al. 2002). 
Tabla 1. Número de especies de mamíferos (> $1 \mathrm{~kg}$ ) objetivo de los cazadores en bosques tropicales de Suramérica, África y Asia e islas asociadas. El número de especies amenazadas se indica entre paréntesis. Datos de Peres (2000), Fa y Peres (2001) y Corlett (2007). Información de las especies amenazadas tomada de UICN (2012). Datos taxonómicos derivados de Duff y Lawson (2004).

\begin{tabular}{l} 
Orden/Géneros \\
CINGULATA \\
Armadillos (Dasypus, Euphractus, Cabassous, Priodontes) \\
\hline LAGOMORPHA
\end{tabular}

\section{LAGOMORPHA}

Conejos (Sylvilagus)

$1(0)$

\section{RODENTIA}

Ardillas, ratas, puercoespines y capibara (Funisciurus, Ratufa, Petaurista, Cricetomys, Rhizomys, Hystrix, Atherurus, Trichys, Thryonomys, Dasyprocta, Cuniculus, Hydrochoerus)

\section{Suramérica}

$5(1)$

\section{CIMOLESTA}

Pangolines (Manis, Smutsia, Phataginus, Uromanis)

\section{CARNIVORA}

Civeta africana de las palmeras (Nandinia)

Ginetas y civetas (Viverra, Genetta, Civettictis, Viverricula, Poina, Prionodon, Macrogalidia, Paguma,

Paradoxurus, Arctictis, Hemigalus, Diplogale)

Osos (Helarctos)

Gatos (Felis, Neofelis, Panthera)

Mangostas (Atilax, Crossarchus, Bdeogale)

Mustélidos (Lutra, Pteronura, Mustela, Aonyx, Melogale, Eira)

\section{CHIROPTERA}

Murciélagos frugívoros (Eidolon, Pteropus, Rousettus)

$7(0)$

$5(0)$

$23(0)$

\section{PRIMATES}

$-$

$3(0)$

$\quad 5(0)$

$12(0)$

$3(1)$

$-$

$2(0)$

$4(2)$

$3(0)$

$4(0)$

$1(0)$

$4(0)$

$10(1)$

$40+$

Prosimios (Galago, Euoticus, Perodicticus, Arctocebus)

Colobos (Colobus, Piliocolobus, Procolobus)

Langures (Presbytis, Trachypithecus, Pygathrix, Nasalis)

Macacos (Macaca)

Dril y mandril (Mandrillus)

Mangabeyes (Cercocebus, Lophocebus)

Cercopitecos (Cercopithecus, Allenopithecus, Miopithecus)

Grandes simios (Pan, Gorilla, Pongo)

Gibones (Hylobates)

Tamarinos/marmosetas (Saguinus, Leontopithecus, Callithrix, Callimico)

Monos ardilla (Saimiri)

Monos capuchinos (Cebus)

Monos titís (Callicebus)

Sakis (Pithecia, Chiropotes, Cacajao)

Monos arañas (Ateles, Brachyteles)

Monos lanudos (Oreonax, Lagothrix)

Monos aulladores (Allouata)

\section{TUBULIDENTATA}

Oricteropo (Orycteropus)

$1(0)$

\section{ARTIODACTYLA}

Cerdos/Pecaríes (Sus, Potamochoerus, Hylochoerus, Babyroussa, Tayassu, Dicotyles)

$2(0)$

$(0)$

$2(0)$

Cérvidos (Muntiacus, Cervus, Mazama)

Bóvidos (Neotragus, Bos, Bubalus, Tragelaphus, Cephalophus, Syncerus, Capricornis, Pseudoryx)
$-$

\section{PERISSODACTYLA}

Rinocerontes (Dicerorhinus, Rhinoceros)

Tapires (Tapirus)

\section{URANOTHERIA}

Damán arborícola (Dendrohyrax)

Elefantes (Loxodonta, Elephas)
$14(0)$

$12(0)$

$\begin{array}{ll}- & 1(0) \\ - & 1(1)\end{array}$


Tabla 2. Número total de especies cazadas por continente con el número de especies amenazadas entre paréntesis.

\begin{tabular}{lcccc}
\hline Grupo & Suramérica & África & Asia & Total \\
\hline Primates & $111(31)$ & $68(18)$ & $69(21)$ & $248(70)$ \\
Ungulados & $4(0)$ & $25(5)$ & $47(0)$ & $76(5)$ \\
Roedores & $7(0)$ & $5(0)$ & $23(0)$ & $35(0)$ \\
Carnívoros & $6(1)$ & $13(0)$ & $21(2)$ & $40(3)$ \\
Murciélagos frugívoros & 0 & $10(1)$ & $40(0)$ & $50(1)$ \\
Otros & $8(2)$ & $6(1)$ & $7(1)$ & $21(4)$ \\
Total & $136(34)$ & $127(25)$ & $\mathbf{2 0 7 ( 2 4 )}$ & $\mathbf{4 7 0 ( 8 3 )}$ \\
\hline
\end{tabular}

(Robinson y Bennett 2002, Polet y Ling 2004), y las presas preferidas por los cazadores incluyen cada vez más especies en peligro de extinción, en lugar de especies comunes para el consumo alimentario. Esto es lo que ocurre, por ejemplo, con tigres, osos y pangolines en el norte de Myanmar (Rao et al. 2005) o los grandes simios en Gabón (Starkey 2004). El uso de una gran variedad de especies silvestres en la medicina tradicional china es particularmente importante, (Nooren y Claridge 2001; Shepherd 2001; Wilkie y Lee 2004) y no tiene parangón en otros países de África o América. Los comerciantes penetran hasta los lugares más remotos en busca de las especies más demandadas (Nijman 2005). En algunas zonas, los cazadores profesionales foráneos son la principal amenaza (por ejemplo, los cazadores vietnamitas en Laos; Nooren y Claridge 2001). El agotamiento de una especie puede dar lugar a su sustitución por otra especie similar (por ejemplo, los huesos de macaco por los de langur). Además, también existe un gran mercado regional de animales vivos que se utilizan como mascotas. Aunque este mercado está dominado por las aves, hay una creciente demanda de cachorros de primates, ardillas y de cualquier otro animal atractivo en estado juvenil (Shepherd et al. 2005).

En las selvas tropicales de África, la información sobre la cantidad de carne de caza objeto de comercio extralocal es relativamente reciente. Las investigaciones previas (King 1994), basadas en datos de pocos cazadores, sostenían que la mayoría de la carne se consumía en el entorno de procedencia. Por el contrario, en un estudio basado en el seguimiento de 42 cazadores (Fa y García Yuste 2001), sólo el $23 \%$ de los animales cazados se destinaron al consumo doméstico, $6 \%$ al sustento in situ de los propios cazadores, mientras que la mayoría de las piezas (68\%) se canalizó a los mercados públicos. La proporción de animales vendidos por cazador era variable, hasta el punto de no existir correlación entre la cantidad vendida y la cantidad cazada. En cualquier caso, independientemente del volumen de piezas abatidas, una gran proporción de los animales se destinaba a la venta.

La carne de caza se come fresca o ahumada, en sopas y guisos, y en menor proporción asada o frita. Un estudio realizado en Ghana por Ntiamoa-Baidu (1998) corroboró que la mayoría de las personas entrevistadas (96\%) consumía la carne en sopas y la cocinaba en su casa. No obstante, el $20 \%$ de los encuestados también lo hacía en restaurantes locales (llamados 'chop bars'). Cowlishaw et al. (2005) destacan la importancia de esos restaurantes locales en la cadena de distribución de carne de caza en Ghana, al igual que constataron East et al. (2005) en Guinea Ecuatorial continental. Los estudios que evalúan la contribución de la carne de caza a las economías domésticas son tan escasos como la evaluación del impacto ecológico que provoca la caza. Esto hace que sea difícil diseñar enfoques de mitigación, ya que todavía no se conoce bien la importancia cuantitativa de la carne de caza en la dieta de los consumidores y en los ingresos domésticos.

La mayoría de los estudios sobre consumo de carne de caza informa acerca de la frecuencia (días/semana) o cantidad (peso/persona) de dicho producto en los hogares locales (por ejemplo, Koppert y Hladik 1990; Koppert et al. 1996; Ntiamoa-Baidu 1998; Starkey 2004; Albretchsen et al. 2006). No obstante, la com- paración entre estos y otros estudios es problemática, porque a menudo está poco claro el tamaño de muestra que se ha empleado en la estimación, así como si las mediciones se basan en carcasas completas o en piezas deshuesadas.

Asumiendo estas limitaciones metodológicas, se han publicado algunas estimaciones de consumo de carne de caza. Los datos ofrecen un amplio rango, desde $0.05 \mathrm{~kg} /$ persona/día hasta 0.28 $\mathrm{kg} /$ persona/día (Chardonnet et al. 1995). Estos datos pueden reflejar diferencias en la dependencia de la población estudiada respecto al consumo de carne versus pescado (ver abajo), así como variaciones imputables al momento del año en que se realizaron los muestreos de campo. Por consiguiente, cabe reiterar que no hay suficiente información en los estudios hasta ahora publicados (por ejemplo, en Ntiamoa-Baidu 1998) para evaluar las posibles fuentes de error. No obstante, es un hecho constatado que, en algunos países de África Central, el consumo de carne por persona y año, la mayor parte de la cual proviene de la caza, es comparable al de los países desarrollados (Fig. 3).

El estudio de Koppert et al (1996) es, probablemente, una de las investigaciones más exhaustivas sobre la dieta estacional de varias poblaciones humanas que viven en los bosques de África ecuatorial y su entorno. El estudio se centró en una serie de grupos étnicos de Camerún, basándose en una amplia muestra de hogares. El alimento vegetal básico de todas las poblaciones estudiadas representaba la principal fuente de energía, pero el pescado y la carne eran la principal fuente de proteínas. Las dietas basadas en raíces y tubérculos, especialmente la mandioca, eran muy bajas en proteínas y otros nutrientes. Esta escasez se suplía con una importante ingesta de proteína animal basada en el pescado y carne de caza. Por lo tanto, los cultivos agrícolas proporcionaban la mayor parte de las calorías a estas comunidades, mientras que la carne era su principal fuente de proteínas.

El estudio citado mostraba que la caza aportaba entre el $70 \%$ y el $88 \%$ de las proteínas ingeridas por los diversos grupos étnicos. Sin embargo, la fuente de proteínas variaba en relación con la proximidad de la población a la costa. Así, los Yassa, que viven en la costa atlántica, pescaban en el mar y cultivaban mandioca, mientras que para los pigmeos Kola, que viven en un bosque climácico, la principal fuente de proteínas era la carne de caza. Cabe concluir que cuando las familias no disponen de recursos alternativos, cual-

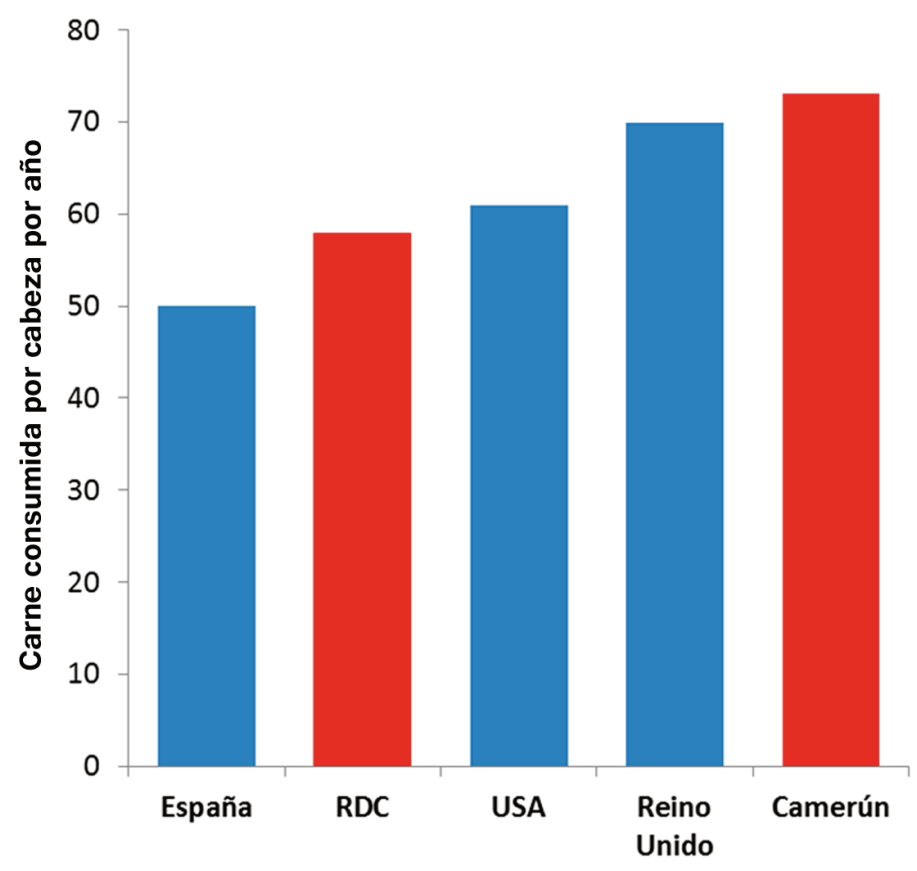

Figura 3. Peso medio de carne (en kilogramos) consumida por persona y año en dos países de África central, comparado con algunos países desarrollados. Datos según Koppert et al. (1996), Wilkie et al. (1998), Regmi (2001) y MAGRAMA (2012). 
quier intento de restringir la caza puede dar lugar a que los niños sufran las consecuencias de una dieta deficitaria en proteínas, lo que implica retrasos en el crecimiento y en el aprendizaje. En la actualidad sólo la pesca y la carne procedente de animales domésticos son sustitutos accesibles como fuente de proteínas. El problema es que la producción de ambas es deficitaria y no constituye una alternativa realista para la caza utilitaria.

Según un estudio realizado por Fa et al. (2005) en los países de la Cuenca del Congo, el aporte de proteínas procedentes de la caza descenderá significativamente si la captura de poblaciones presa continúa al ritmo actual (Cuadro 1), aunque hay diferencias sustanciales entre países (Fa et al. 2002). La República Centroafricana, República Democrática del Congo y la República del Congo muestran una rápida intensificación de la extracción en relación a las tasas de producción, mientras que en Camerún y Gabón los incrementos son mucho más lentos. Esto sugiere que las áreas más críticas se encuentran en la región central de la Cuenca del Congo. En términos de suministro, sólo Gabón es capaz de depender de la carne de caza. El resto de los países tendrá que recurrir a otras fuentes de proteínas procedentes del sector ganadero. Las previsiones indican que incluso si el consumo se redujese hasta niveles sostenibles, las proteínas de origen no cinegético serían insuficiente para cubrir las necesidades de una población humana que actualmente crece por encima del $3 \%$ anual (FAO 2012). La única excepción sería Gabón, ya que este país alberga una densidad de población muy baja (alrededor de una persona por $\mathrm{km}^{2}$ ) y una importante cobertura de bosques relativamente bien conservados (más del $80 \%$ del territorio). Sin embargo, en Camerún y la República Democrática del Congo, por ejemplo, sucede todo lo contrario.

\section{Cuadro 1: Resumen de los resultados de Fa et al. (2003)}

Requerimiento medio recomendado de proteínas diarias (de la Organización Mundial para la Salud - OMS): $52 \mathrm{~g} /$ persona/día.

Suministro actual estimado de proteínas procedentes de carne de caza: $48 \mathrm{~g}$.

Suministro actual de proteínas no procedente de carne de caza: $34 \mathrm{~g}$.

Suministro sostenible de proteínas procedentes de carne de caza: $6.5-13 \mathrm{~g} /$ persona/día (actual).

0.4-0.8 g/persona/día en el año 2050 (teniendo en cuenta la deforestación y el crecimiento de la población).

El suministro total de proteínas descenderá de $85 \mathrm{~g}$ a 41 g/persona/día en el año 2050, debido a la reducción en la disponibilidad de carne de caza.

Esta cifra representa el $79 \%$ del valor mínimo recomendado por la OMS.

Todos los indicadores manejados parecen alertar de que un gran número de especies de mamíferos forestales de mediano y gran tamaño desaparecerá a no muy largo plazo, provocando una inseguridad alimentaria entre los habitantes de la mayor parte de la Cuenca del Congo (Fa et al. 2002). La precisión de estas predicciones depende de la fiabilidad de los datos de extracción utilizados por FAO. Por ello es preciso tener cierta cautela, ya que dichos datos proceden de estudios realizados en diferentes momentos, lo cual puede distorsionar la realidad de los hechos. No obstante, los cálculos de las tasas de extracción para la Cuenca del Congo son aplicables a toda la región, puesto que no existen diferencias étnicas o raciales que determinen un consumo diferencial de carne silvestre por parte de los habitantes de la zona, tal como sucede en otros países africanos (por ejemplo, entre musulmanes y cristianos en Guinea [Ham 1998]).

\section{Conclusiones}

Frente a un pronóstico sombrío, desde el punto de vista de la sostenibilidad biológica, no se puede ignorar que la carne de caza posee gran importancia en la dieta de los habitantes de las zonas de selva tropical del mundo. El duro contraste entre estas dos realidades antagónicas plantea retos insoslayables para el desarrollo de una política coherente de conservación. A la hora de ponderar el valor utilitario que tiene la carne de caza, es necesario tener en cuenta no sólo los niveles absolutos de extracción, sino también los beneficios dietéticos, económicos y sanitarios que proporciona a nivel familiar y social. Por lo tanto, el punto de partida de cualquier discusión institucional sobre el comercio de carne de caza debe sustentarse en una actitud tolerante y comprensiva, en lugar de esgrimir posturas axiomáticas que sólo contemplan los problemas de conservación de especies que subyacen en relación a este tema. No se puede ignorar la amplia aceptación de estas prácticas extractivas ni las utilidades que proporcionan a las sociedades que las practican. Obviamente, tampoco se trata de volver la espalda a las graves amenazas que existen para la conservación de la biodiversidad, y de determinadas especies en particular en muchas de estas áreas, como se ha documentado anteriormente. Pero la falta de apoyo a proyectos de investigación que intentan abordar esta problemática genera una falsa dicotomía, caza versus conservación, dejando al margen la única alternativa razonable que consiste en una conciliación de intereses.

Puesto que el comercio de carne de caza está muy arraigado en las zonas tropicales, integra en armonía a cazadores, vendedores y compradores. En general existen pocas limitaciones para la extracción y venta libre de la mayoría de las especies, y los mercados suelen enfocarse a las clases sociales menos favorecidas. Aunque la información sobre la estructura de los mercados de carne de caza es limitada (fuertes presiones conservacionistas han propiciado un comercio sumergido que inhibe los esfuerzos de investigación), no parece que exista una concentración significativa de las capturas en puntos de venta centralizados. Por ejemplo, abundan las subcontratas de cazadores que no pueden pagar por sí mismos armas y municiones, cuyas capturas abastecen mercados no locales o se exportan. Asimismo, la caza con lazos se utiliza para el autoabastecimiento o para la venta directa sin pasar por los mercados de aldeas y pueblos.

Una parte significativa del valor económico del producto es retenida por el cazador, en mayor medida que otros productos forestales como la madera o cultivos agrícolas (Ntiamou-Baidu 1998; Mendelson et al. 2003; Brown y Williams 2003). A diferencia de la cría de animales domésticos, el esfuerzo de trabajo personal que requiere la caza suele ser discontinuo y fácil de conciliar con el ciclo agrícola. Salvo en los casos en que sea factible la caza a tiempo completo (como en la isla de Bioko a finales de los años 1990 [Fa et al. 2000]), la carne de caza representa una opción complementaria de ingresos por su alto rendimiento y versatilidad. Especialmente para los pequeños agricultores, la caza resulta ser una actividad muy atractiva.

Comerciar con carne de caza es, por tanto, un negocio de bajo riesgo y flexible, compatible con otras actividades que, en conjunto, garantizan un nivel básico de subsistencia y renta a las clases sociales menos pudientes. La tecnología necesaria para la extracción de animales es accesible a cualquier persona, ya que no implica necesariamente la tenencia de armas de fuego y el consumo de munición. De este modo la caza relega a otras opciones más lucrativas pero que requieren una aportación económica inicial (McSweeney 2004 y 2005; De Merode et al. 2003 y 2004), y encaja con las estrategias de subsistencia a la que recurren los pobres, las cuales tienden a ser variadas y exentas de costes de inversión (véase, por ejemplo, Chambers 1987; Carney 1998).

En la mayoría de los casos son los hombres los que cazan y las mujeres las que se encargan del procesamiento y comercialización de las piezas hasta los puntos de venta en los "chop bars" y restaurantes, que suelen ser establecimientos familiares en la escena urbana africana. La carne de caza ahumada tiene excelentes posibilidades de almacenamiento y es compatible con el acopio de productos agrícolas. Por su facilidad de transporte y el alto ratio valor/peso, la carne de caza ha encajado bien en la dinámica de la 
vida rural en los trópicos, sobre todo en escenarios de pobreza. Sin embargo, la posibilidad de seguir manteniendo los niveles actuales de abastecimiento es cada vez más inviable.

Por lo tanto, sería un error asumir que la carne de caza constituye sólo un componente facultativo de escaso valor añadido para la subsistencia y economía de las familias, en cuyo caso sería razonable prohibir su recolección o sustituirla por otras alternativas (Bennett et al, 2007). De acuerdo con lo hasta aquí expuesto, la dependencia actual de las poblaciones humanas respecto a la carne de caza es un conflicto sumamente complicado de resolver. El problema se enmarca dentro de un contexto político más que biológico, no sólo a nivel nacional interno sino también internacional. De hecho, para cubrir las necesidades proteicas de la población humana en países de alta biodiversidad biológica, es necesario contar con la ayuda de organizaciones internacionales como la FAO y la implicación de los países desarrollados. La producción de alimentos alternativos no es la única vía para cambiar la situación actual, ni sería realista incrementar la superficie de producción de dichos alimentos en lugares como la Cuenca del Congo. También es fundamental continuar los esfuerzos para desarrollar una investigación científica práctica y socialmente responsable, con objeto de proteger aquellas especies que están más amenazadas por la caza. En última instancia, es una obligación ética superar la fase puramente descriptiva de la ciencia, porque condena a los científicos a ser meros espectadores de la extinción de especies y del infortunio humano.

\section{Agradecimientos}

Los autores agradecen el apoyo financiero de los fondos FEDER de la Unión Europea y de la Agencia de Obra Pública-Consejería de Obras Públicas y Vivienda, Junta de Andalucía (Proyecto G-GI3000/IDIG).

\section{Referencias}

Albrechtsen, L., Fa, J.E., Barry, B., Macdonald, D.W. 2006. Contrasts in availability and consumption of animal protein in Bioko Island, West Africa: the role of bushmeat. Environmental Conservation 32:340-348.

Bennett, E.L., Rao, M. 2002. Wild meat consumption in Asian tropical forest countries: is this a glimpse of the future for Africa? In: S. Mainka and M. Trivedi (eds.), Links between Biodiversity, Conservation, Livelihoods and Food Security: the Sustainable Use of Wild Species for Meat, pp 39-44. IUCN, Gland, Switzerland and Cambridge, UK.

Bennett, E.L., Robinson, J.G. 2000. Hunting of Wildlife in Tropical Forests: Implications for Biodiversity and Forest Peoples. Global Environment Division, The World Bank, Washington DC, USA.

Bennett, E.L., Blencowe, E., Brandon, K., Brown, D., Burn, R.W., Cowlishaw, G., Davies, G., Dublin, H., Fa, J.E., Milner-Gulland, E.J., Robinson, J.G., Rowcliffe, J.M., Underwood, F.M., Wilkie, D.S. 2007. Hunting for consensus: reconciling bushmeat harvest, conservation, and development policy in West and Central Africa. Conservation Biology 21:884-887.

Brown, D., Williams, A. 2003. The case for bushmeat as a component of development policy: issues and challenges. International Forestry Review 5, 148-155, reproduced in The Earthscan Reader in Forestry and Development, ed. J. Sayer, 2005, pp.193-205.

Carney, D. 1998. Sustainable Rural Livelihoods: What Contribution Can We Make? DFID, London, UK.

Chaber, A.L., Allebone-Webb, S., Lignereux, Y., Cunningham, A.A., Rowcliffe, J.M. 2010.The scale of illegal meat importation from Africa to Europe via Paris. Conservation Letters 3:317-323.

Chambers, R. 1987. Sustainable Livelihoods, Environment and Development: Putting Poor Rural People First. IDS Discussion Paper No. 240, Brighton Sussex, UK.

Chardonnet, P., Fritz, H., Zorzi, N., Feron, E. 1995. Current importance of traditional hunting and major contrasts in wild meat consumption in subSaharan Africa. En: Bissonette, J.A. Krausman, P.R. (eds), Integrating People and Wildlife for a Sustainable Future, pp. 304-307. The Wildlife Society, Bethesda, USA.

Corlett, R.T. 2007. The impact of hunting on the mammalian fauna of tropical Asian forests Biotropica 39:292-303.
Cowlishaw, G., Mendelson, S., Rowcliffe, J.M. 2005. Evidence for post-depletion sustainability in a mature bushmeat market. Journal of Applied Ecology 42:460-468.

De Merode E., Homewood, K., Cowlishaw, G. 2003. Wild resources and livelihoods of poor households in DRC, ODI Wildlife Policy Briefings, No.1, November

De Merode, E., Homewood, K., Cowlishaw, G. 2004. The value of bushmeat and other wild foods to rural households living in extreme poverty in the eastern Democratic Republic of Congo. Biological Conservation 118:573-581.

Duff A., Lawson, A. 2004. Mammals of the World - A Checklist. A \& C Black, London, UK.

Dupain, J., Nackoney, J., Vargas, J. M., Johnson, P. J., Farfán, M. A., Bofaso, M., Fa, J. E. 2012. Bushmeat characteristics vary with catchment conditions in a Congo market. Biological Conservation 146:32-40.

East, T., Kümpel, N.F., Milner-Gulland, E.J., Rowcliffe J.M. 2005. Determinants of urban bushmeat consumption in Río Muni, Equatorial Guinea. Biological Conservation 126:206-215.

Fa, J.E. 2007. Bushmeat markets - white elephants or red herrings? In: Davies, G. y Brown, D. Bushmeat and Livelihoods: Wildlife Management and Poverty Reduction, Blackwells, Oxford. UK.

Fa, J.E., Brown, D. 2009. Impacts of hunting on mammals in African tropical moist forests: a review and synthesis. Mammal Review 39:231-264.

Fa, J.E., García Yuste, J.E. 2001. Commercial bushmeat hunting in the Monte Mitra forests, Equatorial Guinea: extent and impact. Animal Biodiversity and Conservation 24:31-52.

Fa, J.E., Peres, C.A. 2001. Game vertebrate extraction in African and Neotropical forests: an intercontinental comparison. En: Reynolds, J.D., Mace, G.M., Redford, K.H., Robinson, J.G. (eds). Conservation of Exploited Species, pp. 203-241. Cambridge University Press, Cambridge, UK.

Fa, J.E., Garcia Yuste, J.E., Castelo, R. 2000. Bushmeat markets on Bioko Island as a measure of hunting pressure. Conservation Biology 14:16021613.

Fa, J.E., Peres, C.A., Meeuwig, J. 2002. Bushmeat exploitation in tropical forests: an intercontinental comparison. Conservation Biology 16:232237.

Fa, J.E., Currie, D., Meeuwig, J. 2003. Bushmeat and food security in the Congo Basin: linkages between wildlife and people's future. Environmental Conservation 30:71-78.

Fa, J.E., Ryan, S.L., Bell, D.J. 2005. Hunting vulnerability, ecological characteristics and harvest rates of bushmeat species in afrotropical forests. Biological Conservation 121:167-176

Fa, J.E., Seymour, S., Dupain, J., Amin, R., Albrechtsen, L., Macdonald D. 2006. Getting to grips with the magnitude of exploitation: Bushmeat in the Cross-Sanaga rivers region, Nigeria and Cameroon. Biological Conservation 129:497-510.

FAO 2012. Food and Agriculture Organization of the United Nations statistical databases [www document]. URL http://apps.fao.org/

Ham, R. 1998. Nationwide Chimpanzee Census and Large Mammal Survey: Republic of Guinea. Brussels, Belgium: European Union.

Hart, J.A. 2000. Impact and sustainability of indigenous hunting in the Ituri Forest, Congo-Zaire: a comparison of hunted and unhunted duiker populations. In J.G. Robinson \& E.L. Bennett (eds.) Hunting for Sustainability in Tropical Forests, pp 106-153. Columbia University Press, New York, USA.

King, S. 1994. Utilisation of Wildlife in Bakossiland, West Cameroon with particular reference to primates. TRAFFIC Bulletin 14:63-73.

Koppert, G., Dounias, E., Froment A., Pasquet, P. 1996. Food consumption in three forest populations of the southern coastal area of Cameroon: Yassa - Mvae - Bakola. En: Hladik, C.M., Linares, O.F., Pagezy, H., Semple, A., Hadley, M. (eds.), Tropical forests, people and food: Biocultural interactions and applications to development, pp. 295-310. UNESCO, Paris, France.

Koppert, G., Hladik, C.M. 1990. Measuring food consumption. En: Hladik, C.M., Bahuchet, S., Garine, I. (eds). Food and Nutrition in the African Rain Forest, pp. 59-63. Paris, France. UNESCO/CNRS.

Li, Y., Li. D.M. 1998. The dynamics of trade in live wildlife across the Guangxi border between China and Vietnam during 1993-1996 and its control strategies. Biodiversity and Conservation 7:895-914.

Li, W., Wang, H. 1998. Wildlife trade in Yunnan Province, China, at the border with Vietnam. TRAFFIC Bulletin 18:21-30. 
McSweeney, K. 2004. Forest product sale as natural insurance. Society and Natural Resources 17:39-56.

McSweeney, K. 2005. Forest product sale as financial insurance: evidence from Honduran smallholders. ODI Wildlife Policy Briefing No. 10. Overseas Development Institute, London, UK.

Mendelson, S., Cowlishaw, G., Rowcliffe, J.M. 2003. Anatomy of a bushmeat commodity chain in Takoradi, Ghana. The Journal of Peasant Studies 31:73-100

Ministerio de Agricultura, Alimentación y Medio Ambiente (MAGRAMA) 2012. La alimentación en España. Resumen de los datos más relevantes. MAGRAMA, Madrid. España.

http://www.magrama.gob.es/es/alimentacion/temas/consumo-y-comercializacion-y-distribucion-alimentaria/panel-de-consumo-alimentario/resumen-anual-de-la-alimentacion/default.aspx

Nijman, V. 2005. In full swing: An assessment of trade in orang-utans and gibbons on Java and Bali, Indonesia. TRAFFIC Southeast Asia, Petaling Jaya, Malaysia.

Nooren, H., Claridge, G. 2001. Wildlife trade in Laos: The end of the game. Netherlands Committee for IUCN, Amsterdam, The Netherlands.

Ntiamoa-Baidu, Y. 1998. Wildlife and Food Security in Africa. FAO Conservation Guide 33. Rome, Italy: Food and Agriculture Organization of the United Nations.

Pauly, D., M.L. Palomares. 2005. Fishing down marine food webs: it is far more pervasive than we thought. Bulletin of Marine Science 76:197211.

Peres, C.A. 2000. Effects of subsistence hunting on vertebrate community structure in Amazonian forests. Conservation Biology 14:240-253.

Peres, C.A. 2001. Synergistic effects of subsistence hunting and habitat fragmentation on Amazonian forest vertebrates. Conservation Biology 15:1490-1505.

Polet, G., Ling, S. 2004. Protecting mammal diversity: Opportunities and constraints for pragmatic conservation management in Cat Tien National Park, Vietnam. Oryx 38:186-196.

Rabinowitz, A. 1995. Helping a species go extinct: The Sumatran Rhino in Borneo. Conservation Biology 9:482-488.
Rao, M.H., Myint, T., Zaw, T., Htun, S. 2005. Hunting patterns in tropical forests adjoining the Hkakaborazi National Park, north Myanmar. Oryx 39:292-300.

Redford, K. 1992. The empty forest. BioScience 42:412-422.

Redford, K., Robinson, J. 1987. The game of choice: patterns of Indian and colonist hunting in the Neotropics. American Anthropologist 89:650-667.

Regmi, A., ed. 2001.Changing Structure of Global Food Consumption and Trade. ERS, USDA, Agriculture and Trade Report WRS-01-1.

Robinson, J., Bennett, E.L. 2002. Will alleviating poverty solve the bushmeat crisis? Oryx 36:332.

Robinson, J.G., Bennett, E.L. 2004. Having your wildlife and eating it too: an analysis of hunting sustainability across tropical ecosystems. Animal Conservation 7:397-408.

Shepherd, C.R. 2001. Observations on wildlife trade at Golden Rock, Myanmar. TRAFFIC Bulletin 19:7-10.

Shepherd, C.R., Sukumaran, J., Wich, S.A. 2005. Open season: An analysis of the pet trade in Medan, Sumatra 1997-2001. TRAFFIC Southeast Asia, Petaling Jaya, Malaysia.

Starkey, M.P. 2004. Commerce and subsistence: the hunting, sale and consumption of bushmeat in Gabon. PhD thesis, University of Cambridge, Cambridge.

UICN 2012. The IUCN Red List of Threatened Species. Version 2012.2. <http://www.iucnredlist.org>. Consultado el 23 enero 2013.

Wilkie, D.S., Lee, R.J. 2004. Hunting in agroforestry systems and landscapes: Conservation implications in West-Central Africa and Southeast Asia. In G. Schroth, G. A. B. da Fonseca, C. A. Harvey, C. Gascon, H. L. Vasconcelos y A.-M. N. Izac (Eds.). Agroforestry and biodiversity conservation in tropical landscapes, pp. 346-370. Island Press, Washington, DC, USA.

Wilkie, D.S., Curran, B., Tshombe, R., Morelli, G.A. 1998. Modeling the sustainability of subsistence farming and hunting in the Ituri Forest of Zaire. Conservation Biology 12:137-147.

Wilkie, D.S., Carpenter, J.F. 1999. Bushmeat hunting in the Congo Basin: an assessment of impacts and options for mitigation. Biodiversity and Conservation 8:927-955. 